\title{
PHYSIOLOGY AND PATHOPHYSIOLOGY OF THERMOREGULATION IN THE NEONATE
}

\author{
Singer D \\ Dept. of Neonatology and Pediatric Intensive Care Medicine, \\ University Medical Center Eppendorf, Hamburg, Germany \\ dsinger@uke.de
}

\begin{abstract}
In view of the adverse effects of hypothermia on term and preterm babies, heat therapy is of utmost importance in neonatology. However, despite considerable technical progress in the field, some fundamental physical and physiological questions remain to be answered.
\end{abstract}

Keywords: Human neonates, body temperature, hypothermia, heat therapy, metabolic rate

\section{Term Neonates}

Contrary to what is often assumed, term neonates do not have an "immature" thermoregulation, but are well adapted to their large surface-to-volume ratio by an elevated basal metabolic rate and by the capability to produce extra heat in the brown adipose tissue. Due to the invisibility of this so-called non-shivering thermogenesis (NST), they may experience an undetected thermal stress at ambient temperatures that are still taken for comfortable by their adult caregivers. Although deliberate (induced) cooling is now regarded as a therapeutic option in cases of perinatal asphyxia, inadvertent (accidental) hypothermia may result in a gap between $\mathrm{O}_{2}$ consumption and supply and itself lead into a "vicious circle" of hypoxia [1].

\section{Preterm Neonates}

Preterm neonates are at even higher risk of hypothermia, due to their smaller body size and to the "true" immaturity of effector systems (lack of isolating white and heatproducing brown adipose tissue). Moreover, they exhibit an increased permeability of the skin (resulting in evaporative heat loss) and a delayed increase in basal metabolic rate (up to the level to be expected from body size). This explains their long-lasting thermal instability which contributes to adverse outcomes such as an increased rate of intracranial hemorrhage [2].

\section{Heat Therapy and Controversial Issues}

To prevent hypothermia and its detrimental effects, heat therapy plays a prominent role in delivery room resuscitation as well as in critical care of term and preterm neonates. Whereas in the delivery room, radiant heaters are the most common source of heat, in the neonatal intensive care unit, both radiant heaters and humidified incubators are currently in use, depending on local and national preferences. As is shown by basic simulation experiments, both are similarly effective, with radiators (preferably enhanced by a plastic sheet spread over the baby to mini- mize evaporative heat loss) doing better than $d r y$ incubators, but humidified incubators outperforming radiators.

With respect to radiant heaters, there is some debate on the desirable infrared (IR) spectrum: Most commercial devices emit long-wave IR-B/-C radiation which, due to its low depth of penetration, bears the risk of superficial burns. However, the introduction of short-wave (waterfiltered) IR-A radiation which offers a better "depth effect" with less surface heating [3], has brought about concerns about presumed ocular risks.

With respect to incubator care, the best way to monitor the baby's heat balance is still controversial: Current techniques include digital rectal thermometers, local skin sensors (attached to the baby's back or belly), and a continuous monitoring of the gradient between central and peripheral temperatures which has the benefit - or drawback - to be superimposed by hemodynamic changes.

\section{Unanswered Questions and Future Prospects}

In contrast to other physiological parameters such as $\mathrm{O}_{2}$ saturation or $\mathrm{CO}_{2}$ tension, the "appropriate" body temperature has so far attracted surprisingly little interest in neonatology although the human fetus is known to be "brooded" at (intrauterine) temperatures near $38^{\circ} \mathrm{C}$. This is clearly higher than targeted in most neonatal intensive care units and might have an underappreciated effect on the (neural) development of preterm neonates.

Beyond the aspect of "physiological appropriateness", the feedback control of modern intensive care incubators encompasses "calorimetric information" on the baby's metabolic rate that might be read out and displayed to the user. This could help adjust the $\mathrm{O}_{2}$ and nutrient supply to the actual demand. First attempts to recalculate the metabolic rate, based on signals from miniaturized heat flow detectors, yielded encouraging results [4].

\section{Bibliography}

[1] M. Gekle, D. Singer, „Temperaturregulation und Wärmehaushalt", in Physiologie, $6^{\text {th }}$ ed. (R. Klinke, H.-C. Pape, A. Kurtz and S. Silbernagl, eds.), ch. 13, pp. 500514, Stuttgart, New York: Thieme, 2010

[2] A. Okken, J. Koch, eds., Thermoregulation of sick and low birth weight neonates, Berlin: Springer, 1995

[3] Singer, D., Schröder, M., Harms, K.: Vorteile der wassergefilterten gegenüber herkömmlicher InfrarotStrahlung in der Neonatologie, Z. Geburtsh. Neonatol., vol. 204, pp. 85-92, 2000

[4] J. Röttgers, M.D. Thesis, Hamburg: in preparation 